\title{
ORIENTABLE SURFACES IN FOUR-SPACE
}

\author{
BY HERMAN GLUCK ${ }^{1}$
}

Communicated by A. W. Tucker, June 23, 1961

1. Introduction. Fränkl and Pontrjagin [1] and Seifert [2] have shown that for any given family of disjoint polyhedral simple closed curves in three-space, there can always be found a polyhedral orientable surface in three-space whose boundary consists precisely of the given curves. The following theorem extends this result to surfaces in four-space.

Theorem 1. Let $M^{2}$ be a locally flat, polyhedral, closed orientable surface (not necessarily connected) in Euclidean four-space, $R^{4}$. Then there is an orientable polyhedral three-manifold, $M^{3}$, in $R^{4}$, whose boundary is $M^{2}$.

Local flatness means that for each vertex $v$ of $M^{2}$, the link of $v$ on $M^{2}$ (a simple closed curve) is unknotted in the link of $v$ in $R^{4}$ (a three-sphere). This condition is purely local and absolutely necessary. On the other hand, the restriction to orientable surfaces is required by the nature of the proof, and I do not know whether nonorientable surfaces of even characteristic in four-space bound nonorientable three-manifolds in four-space. ${ }^{2}$

2. Outline of the proof. $M^{2}$ is first deformed so that its intersections with the horizontal hyperplanes $R_{t}^{3}=\left\{\left(x_{1}, x_{2}, x_{3}, x_{4}\right): x_{4}=t\right\}$ are as simple as possible. What we have in mind is to find orientable surfaces in the $R_{t}^{3}$ whose boundaries are precisely $M^{2} \cap R_{t}^{3}$, in such a continuous way that when considered together they form an orientable threemanifold $M^{3}$ whose boundary is $M^{2}$. The process is carried out with decreasing $t$, and the local flatness of $M^{2}$ assures us that the construction can be begun. As $t$ decreases, $M^{2} \cap R_{t}^{3}$ changes isotopically, except at a finite number of singular values of $t$. A slight deformation of $M^{2}$ insures that we need only consider hyperbolic transformations, in which two arcs come together at a midpoint and then separate like the cross-sections of a saddle surface, and elliptic transformations, in which a simple closed curve shrinks to a point and then disappears (or vice versa). In the hyperbolic case, these arcs already form part

1 The author is a National Science Foundation Predoctoral Fellow and wishes to thank Professor Ralph Fox for his help in the preparation of this paper. The detailed arguments are contained in the author's thesis.

2 Added in proof. This case is considered in a forthcoming paper. 
of the boundary of a cross-sectional surface and the hyperbolic transformation could be extended to the surface in the natural way, except for the possibility of a number of sheets of the surface being in the way. These sheets are simply pierced one after the other with decreasing $t$, the cuts being joined as in [3, page 4 ], to preserve orientability of the cross-sections. Finally, when no more sheets are in the way, the original hyperbolic transformation is extended to the crosssectional surface. That this final transformation does not destroy the orientability of the cross-sections requires a special argument.

In the case of the elliptic transformations, if with decreasing $t$ a point opens up into a simple closed curve (which must be unknotted by the local flatness of $M^{2}$ ), then we simply introduce another component of the cross-sectional surface which with decreasing $t$ opens up from a point into a two-cell. The serious case occurs when a component of $M^{2} \cap R_{t}^{3}$ shrinks to a point and then disappears with decreasing $t$. Call this component $c_{1}$, and let $c_{2}, \cdots, c_{k}$ be the other boundary curves of the component $G$ of the cross-sectional surface containing $c_{1}$. Since $c_{1}$ is unknotted by the local flatness of $M^{2}$, let $D$ be a polyhedral two-cell in $R_{t}^{3}$ bounded by $c_{1}$. Let $c^{\prime}$ be a simple closed curve on $G$ lying in a small neighborhood of $c_{1}$ and "parallel" to $c_{1}$. Because the cross-sectional surface, and hence $G$ is orientable, the linking number of $c^{\prime}$ with $c_{1}$ is the same as the sum of the linking numbers of the $c_{2}, \cdots, c_{k}$ with $c_{1}$. But each of these linking numbers is zero, since $c_{1}$ is about to shrink to a point away from all these curves. Because the linking number of $c^{\prime}$ with $c_{1}$ is zero, the crosssectional surface can be deformed so that a small neighborhood of $c_{1}$ on $G$ meets $D$ only at $c_{1}$, while the total intersection of the crosssectional surface with $D$ consists of a number of simple closed curves. Each of these intersections can be removed by standard hyperbolic transformations with decreasing $t$, until finally $D$ meets the crosssectional surface only at its boundary curve $c_{1}$. By a slight deformation of $M^{2}$, the original elliptic transformation can be altered so as to shrink $c_{1}$ to a point along $D$, closing up a component of the crosssectional surface and completing the construction for the elliptic transformation. When finally $t$ has decreased below the minimum value attained by the fourth coordinates of points of $M^{2}$, the crosssectional surface consists of a number of closed orientable surfaces in a three-dimensional hyperplane $R_{t}^{3}$. It remains to shrink off the components of this surface to points with decreasing $t$. If the resulting $M^{3}$ is to be a manifold, this must be done by first changing these components into two-spheres. But R. H. Fox has shown in $[4$, Theorem 2] that whenever we are given a number of polyhedral 
closed orientable surfaces in three-space, not all of which are twospheres, a hyperbolic transformation may be found which either decreases the total genus or else increases the number of components with positive genus while leaving the total genus unaltered. We carry out such a transformation with decreasing $t$, and repeat the procedure until all the components of the cross-sectional surface are twospheres, which may then be shrunk to points as $t$ decreases further, completing the construction of $M^{3}$.

As the various transformations undergone by the cross-sectional surfaces are topologically equivalent to those experienced by a crosssection of a hypersurface in $R^{4}$ (with due regard being taken of the fact that $M^{3}$ has a boundary), it is easily seen that $M^{3}$ is a manifold. Furthermore, since the cross-sections are orientable and the various transformations preserve orientations, $M^{3}$ is also orientable.

R. H. Bing has pointed out to me that if we are willing to allow a three-dimensional cross-section, then the argument can be completed as soon as the cross-sectional surface becomes closed, for every closed surface in three-space, whether connected or not, is the boundary of a three-dimensional region.

\section{REFERENCES}

1. F. Fränkl and L. Pontrjagin, Ein Knotensatz mit Anwendung auf die Dimensionstheorie, Math. Ann. vol. 102 (1930) pp. 785-789.

2. H. Seifert, Über das Geschlecht von Knoten, Math. Ann. vol. 110 (1934) pp. 571592.

3. C. D. Papakyriakopoulos, On Dehn's lemma and the asphericity of knots, Ann. of Math. vol. 66 (1957) pp. 1-26.

4. R. H. Fox, On the imbedding of polyhedra in 3-space, Ann. of Math. vol. 49 (1948) pp. 462-470.

PRINCETON University 